\title{
Circuit Design of Clos-based On-Chip Interconnection Networks
}

\author{
Yikun Jiang and Mei Yang \\ Department of Electrical and Computer Engineering \\ University of Nevada, Las Vegas, NV 89154 \\ Emails: jiangy3@unlv.nevada.edu, mei.yang@unlv.edu
}

\begin{abstract}
Single-hop non-blocking networks have the advantage of providing uniform latency and throughput, which is important for cache-coherent network-on-chip systems. This paper focuses on high performance circuit designs of multi-stage non-blocking networks as alternatives to crossbars. Existing work shows that Benes networks have much lower transistor count and smaller circuit area but longer delay than crossbars. To reduce the timing delay, we propose to design the Clos network built with larger size switches. Using less than half number of stages than the Benes network, the Clos network with $4 \times 4$ switches can significantly reduce the timing delay. The circuit designs of both Benes and Clos networks in different sizes are conducted considering two types of implementation of the configurable switch: with NMOS transistors only and full transmission gates (TGs). The layout and simulation results under $45 \mathrm{~nm}$ technology show that the TG-based implementation demonstrates much better signal integrity than its counterpart. Clos networks achieve average $60 \%$ lower timing delay than Benes networks with even smaller area and power consumption.
\end{abstract}

Keywords-Crossbars; Benes; Clos; Network-on-Chip (NoC); Schematic; Layout.

\section{Introduction}

Networks-on-Chip (NoCs) have emerged as the key onchip communication architecture for multiprocessor systemson-chip and chip multiprocessors [1][27][29]. Achieving scaling performance for future many-core systems will require high-performance, yet energy-efficient on-chip interconnection networks [2][27][28]. Existing NoC topologies can be classified into two categories: (1) Multi-hop interconnection networks, like mesh [4], torus, concentrated mesh [3], etc., (2) Single-hop non-blocking indirect networks, like crossbar [25], Benes [26], Clos [19], etc.

NoC systems, such as the Tilera Tile64 [5], utilize a distributed mesh-based network to avoid the scaling issues of long wires. However, this improved scalability comes at the expense of nonuniform cache access (NUCA) latencies [15] and high variability in memory access latencies [6], as well as increased design complexity to guarantee correctness and fairness. The study in [14] shows that mesh network's accepted throughput at any given node is highly dependent on the location of the destination node, as shown in Fig. 1. A number of solutions have been developed to solve the problem but at the cost of more complexity routing algorithms and adoption of additional buffers at each router. These buffers

This work was supported in part by NSF under grant no. CNS-1126688. consume significant power and area. According to Intel's projections, the interconnection network itself consumes more than $30 \%$ of total chip power [16][30].
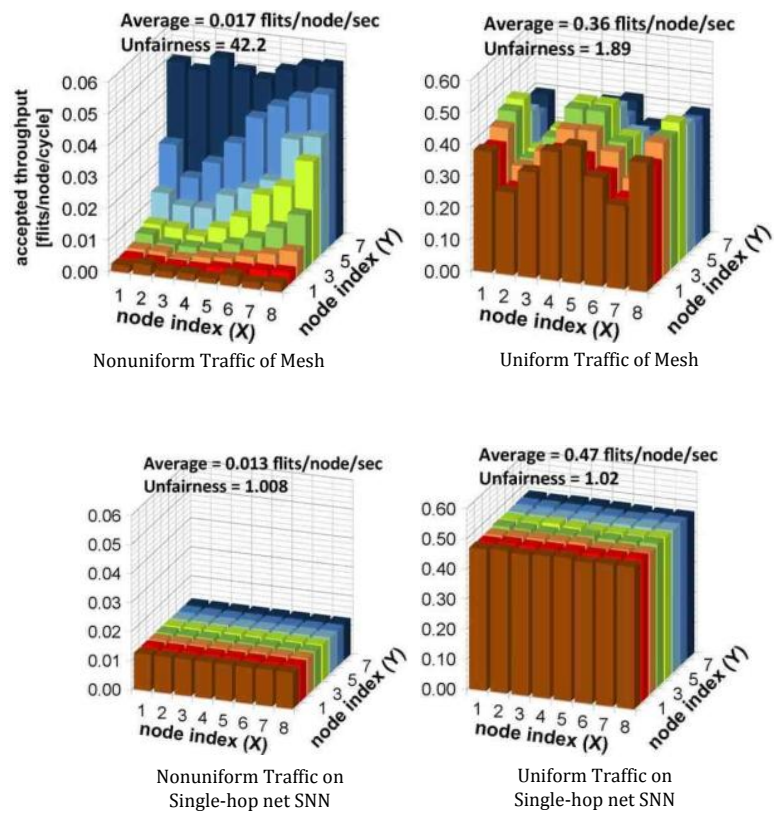

Fig. 1. Traffic analysis of mesh- and crossbar-based NoC [14].

Contrastingly, single-hop non-blocking networks eliminate the need of intermediate buffers thus can provide uniform latency and throughput, which are very important for cachecoherent many-core systems [14][24]. Additionally, due to their high bisection bandwidth, non-blocking networks can potentially provide lower complexity solutions with qualityof-service guarantees than multi-hop networks [16][25]. The crossbar-based swizzle-switch network (SSN) achieves significant performance improvement in throughput $(21 \%)$, cache miss latency (3.0x), and energy savings (25\%) than the mesh-based network [14].

Our study is focused on high performance circuit designs of on-chip non-blocking networks, including single-stage networks (i.e., crossbar) and multi-stage networks (Benes and Clos networks). The scalability of crossbar designs is limited by the quadratically increased circuit complexity. As an alternative to crossbars, Benes networks have much lower transistor count and smaller circuit area but longer delay than crossbars [17]. In [20], 3D folded designs of Benes networks 
and Clos networks built with $2 \times 2$ switches are presented. But there is no exploration of Clos networks with larger size switches. In addition, the aforementioned designs are conducted under $130 \mathrm{~nm}$ or older technology. There is a need to evaluate these network designs under current newer technology.

To reduce the timing delay, we propose to design the Clos network built with larger size switches. Using less than half number of stages than the Benes network, the Clos network with $4 \times 4$ switches can significantly reduce the timing delay. The circuit designs of both Benes and Clos networks in different sizes are conducted considering two types of implementation of the configurable switch: with NMOS transistors only and full transmission gates (TGs). The layout and simulation results under $45 \mathrm{~nm}$ technology show that Clos networks achieve average significant lower timing delay than Benes networks with even smaller area and power consumption.

The rest of the paper is organized as follows. Section 2 reviews existing work on circuit designs of single-hop nonblocking networks. Section 3 describes the properties of nonblocking networks. Section 4 presents the circuit design methodology. Section 5 presents the simulation results. Section 6 concludes the paper.

\section{Related Work}

\subsection{Crossbar Designs}

To provide better bandwidth, Crossbars are used to replace bus-based interconnect fabrics in early multi-core systems, like in the Niagrara2 [7] and IBM BlueGene [13]. Crossbarbased architectures not only can provide the uniform memory access latency that is unachievable in multi-stage NoC systems, but also can potentially provide higher bisection bandwidth and lower complexity solutions for quality-ofservice guarantees than NoC designs. Despite these advantages, large crossbars are generally considered infeasible because the area and power of traditional matrix-style crossbars grow quadratically with crossbar radix [8]. Therefore, it is commonly believed that they become overly expensive for radixes above 32 or 64 [9]. In some work, the crosspoint queueing (CQ) was adopted to replace the traditional input queueing (IQ) to simplify complexity and improve the performance of crossbar scheduling [10]. However, crosspoint queueing was found expensive due to the high partitioning of the switch memory; since there is one memory per crosspoint, the total number of memories grows as $O\left(N^{2}\right)$, which is costly for flow and congestion control algorithms [11][12]. Furthermore, the work in [10] proposed the hierarchically-queued crossbar (HQ) as an organization that lowers memory partitioning. In this organization, an $N \times N$ crossbar is partitioned in $(N / k)^{2} k \times k$ sub-crossbars and memories are placed only at the inputs and outputs of the sub-crossbars. Hence, the total number of memories is reduced from $O\left(N^{2}\right)$ to $O\left(N^{2} / k\right)$. Unfortunately, this organization has a major disadvantage: although partitioning is lowered, it remains unacceptably high, especially when $N$ is large. The reason is that each sub-crossbar has to be relatively small in order to be efficiently scheduled, which, in turn, implies a small $k$ and a quick growth rate of total number of memories.

Existing crossbar circuits mainly adopt MUX-based designs and matrix-based designs [17]. For MUX-based crossbar designs, the latency experienced by a signal depends on crossbar size. For data paths constructed using 2-to-1 multiplexers, doubling the number of inputs results in an additional multiplexer on each line [17]. Pipelined crossbars are proposed to speed up MUX-based designs. Both IBM C64 [18] crossbar and 128x128 crossbar [22] are pipelined MUXbased designs. The IBM Cyclops64 is a 96x96 96-bit-wide crossbar implemented in a $90 \mathrm{~nm}$ technology, running at $533 \mathrm{MHz}$, and occupying $27 \mathrm{~mm}^{2}$, including the circuits for queueing, arbitration, and flow control [18]. In [22], a $128 \times 128$ 32-bit-wide crossbar switch is implemented in $90 \mathrm{~nm}$ CMOS standard-cell ASIC technology. The crossbar operates at $750 \mathrm{MHz}$ and provides a port capacity above $20 \mathrm{~Gb} / \mathrm{s}$, while fitting in a silicon area as small as $6.6 \mathrm{~mm}^{2}$ by filling it at $90 \%$ level (control not included). Though the throughput is dramatically improved with pipelined crossbar designs, the port-to-port latency is kept undesirable.

The complex wire interleaving in traditional MUX-based crossbars causes the layout challenge at high bus widths [14]. Most recent crossbars use matrix-style structures. The results in [17] show that for the same size crossbar, compared with the MUX-based design, the matrix-based design reduces the transistor count by $90 \%$ and latency up to $50 \%$. Conventional matrix-based crossbars consist of the switching fabric and a separate arbiter that configures the crossbar. This decoupled approach imposes the routing challenge and complexity in arbiter design when the radix of the crossbar increases. Passa's work proposed a novel microarchitecture that inverts the locality of wires by orthogonally interleaving the input with the output arbiters, thus reducing the routing area from $O\left(N^{4}\right)$ to $O\left(N^{2} \log ^{2} N\right)$. However, the prohibitive overhead of the arbiter (consuming $60 \%$ of total crossbar area) still limits the design to scale when implementing high-radix crossbar.

\subsection{Benes/Clos Designs}

Though matrix-based crossbars overbeat MUX-based designs in both area and timing delay [17], their scalability is still limited by the quadratically increased circuit complexity. As an alternative to crossbars, Benes networks have much lower transistor count and smaller circuit area but longer delay than crossbars [17]. The circuit design of 2D Benes network shows that the Benes network significantly reduces the transistor count and power consumption compared with the same size matrix-based crossbar design. While the latency result of the Benes network is worse than that of the matrixbased crossbar counterpart [17]. In [20], 3D folded designs of Benes networks and Clos networks built with $2 \times 2$ switches are presented. The numerical analysis shows that $3 \mathrm{D}$ folded design can help improving the latency result. But this work only provides the 3D folded design in theoretical aspect, there is no actual layout of the circuit. As a matter of fact, the TSVs cannot satisfy the density requirement of high-radix crossbar 
connection because each TSV needs a large pad area to guarantee the quality. Besides, in this work there is no exploration of Clos networks with larger size switches.

Clos networks have been adopted to interconnect multi/many cores [16][30] in 2D and 3D structures. However, to the best of our knowledge, there is no work on using the Clos network as a replacement of crossbars.

In the literature, the studies on circuit design of crossbar and Benes/Clos networks are limited. Due to the lack of appropriate wire models, it's very inaccurate to conduct circuit level simulation for designs, which completely neglects any effect that physical routing might have on circuit performance. The transistor level simulation might yield important information about the behavior of the actual physical circuits. However, there seems to be few studies based on transistor level simulation of these network circuits although delay boundaries in terms of number of switches and path length have been established.

In [17], the transistor level circuit designs of matrix/MUXbased crossbars and Benes network are accomplished. But from the layout view presented by the author, the following problems are observed: 1) the transistor level layout design is not optimized enough; 2) the maximum size of the design is limited to $16 \times 16$, which cannot provide enough results to justify the trend for larger size crossbars. In addition, their aforementioned designs are conducted under $130 \mathrm{~nm}$ or older technology. There is a need to conduct transistor level circuit designs of these networks under current newer technology.

\section{Non-Blocking Networks}

The major performance metrics of the circuit designs of non-blocking networks include timing delay, area, and power consumption. The number of stages of a network is the key factor determining the timing delay of the network. Generally, for networks built with the same type of logic units, more stages means longer timing delay. The determining factor of area and power consumption is the transistor count. In this section, we describe the properties of three types of nonblocking networks that are the determining factors of their performance.

\subsection{Crossbar}

The crossbar is a strictly non-blocking network, i.e., any permutation of inputs and outputs can be realized without confliction. As shown in Fig. 2, each input port is connected to each output port through a dedicated logic unit, which is composed of one configurable switch, the basic component used in our circuit design. The number of logic units needed for an $N \times N$ crossbar is $N^{2}$.

The number of stages traversed from one input port to one output port is only one. However, the circuit complexity of crossbars increases quadratically with the crossbar's size. The resulted high power consumption and die area limits the use of crossbars for large-scale NoCs.

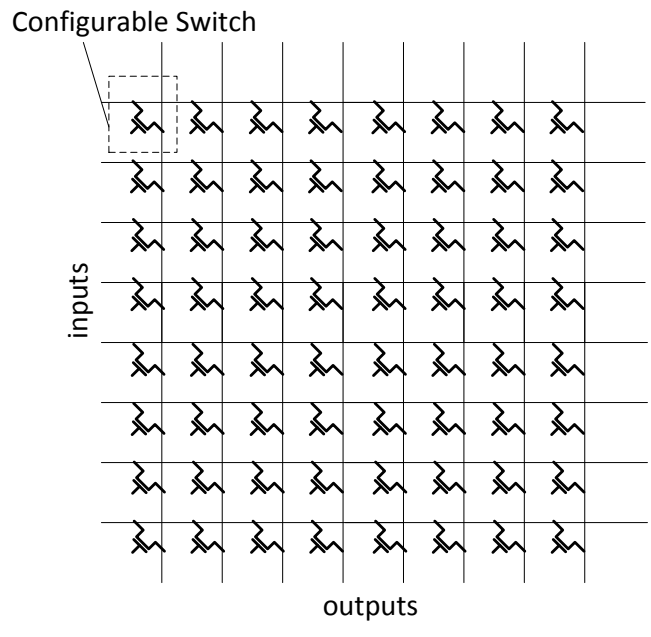

Fig. 2. 8x8 Crossbar

Contrastingly, multi-stage non-blocking networks mitigate the complexity in logic units with rearrangeable non-blocking capability.

\subsection{Benes Network}

An $N \times N$ Benes network basically is built with two symmetrical $N \times N$ butterfly networks. Larger size Benes networks can be built with smaller Benes networks recursively. The basic logic unit is a $2 \times 2$ crossbar switch. As shown in Fig. 3.

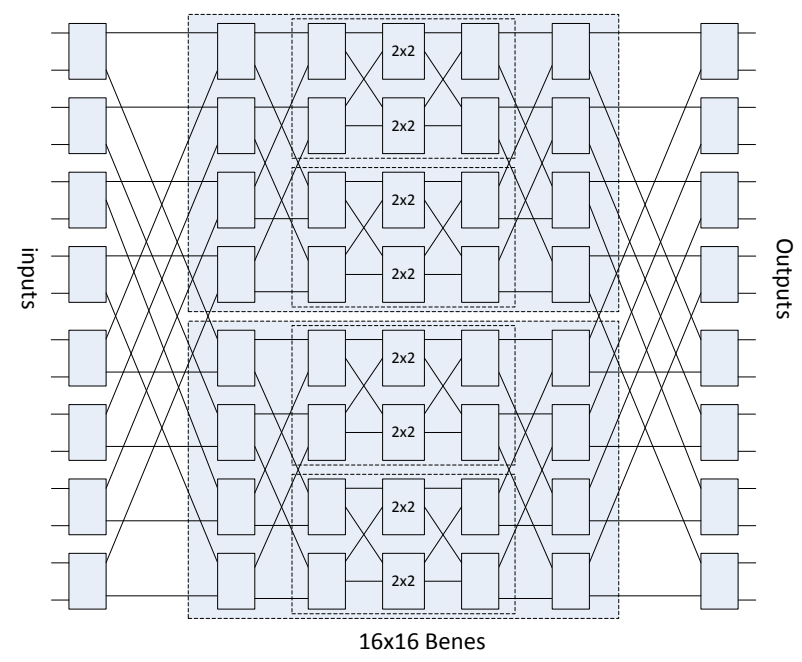

Fig. 3. Benes network

Base on this recursive nature, the number of logic units used in $N \mathrm{x} N$ Benes network can be derived as:

$$
\begin{gathered}
\left\{\begin{array}{c}
f(N)=N+2 f(N / 2) ; \\
f(2)=1 ;
\end{array}=>\right. \\
f(N)=N \log _{2}^{N}-\frac{N}{2}
\end{gathered}
$$

Equation(1) shows the amount of logic units used in a Benes network is significantly reduced compared to the amount of logic units used in the same size crossbar. 
In Benes networks, the number of stages traversed from an input port to an output port increases as the network size increases. The relation between the number of stages of a Benes network and the network size is derived below.

$$
s(N)=2 \log _{2}^{N}-1
$$

Though $O\left(\log _{2}^{N}\right)$ is a slow increasing function of $N$, it is desirable to reduce the number of stages.

\subsection{Clos Network}

To reduce the number of stages for the same size Benes network, we consider Clos networks [22]. A Clos network is composed of three stages of crossbar switches: the input stage, middle stage and the output stage. Each stage is made of a number of same size crossbar switches. A Clos network is defined with a triplet $(m, n, r)$, where $m$ represents the number of switches at the middle stage, $n$ represents the number of input (resp. output) ports of each switch at the input (resp. output) stage, and $r$ is the number of switches at input/output stages. Each input stage crossbar switch has $m$ outputs, each connecting to one of the middle stage switches.

Based on the definition of the Clos network, Fig. 4 shows the semi-recursive Clos networks built with $4 \times 4$ and $2 \times 2$ crossbar switches. Fig. 5(a) and (b) show the structures of the two size switches made by crossbar. The number of logic units (i.e., 2x2/4x4 switches) used in such Clos network is derived as:

$$
\left\{\begin{array}{l}
f(N)=\frac{N}{2}+4 f\left(\frac{N}{4}\right) ;=> \\
f(2)=1, f(4)=1
\end{array}\right.
$$

$f(N)=\left\{\begin{array}{l}\frac{N}{2}\left(\log _{4}^{N}\right)-\frac{N}{4} ; \text { when } \log _{4}^{N} \text { is an integer } \\ \frac{N}{2}\left(\log _{4}^{2 N}\right) ; \text { when } \log _{4}^{N} \text { is not an integer }\end{array}\right.$

Equation (3) shows the number of logic units needed for Clos networks is much smaller than the number of logic units needed by Benes networks. Notice that in (3), when $\log _{4}^{N}$ is not integer, like $N=8,32128, \ldots$, the most middle stage is composed of $2 \times 2$ switches. Fig. 4(b) and (d) show this type of Clos network.

The relation between the number of stages of a Clos network and the network size is shown in Equation (4).

$S(N)=\left\{\begin{array}{l}2\left(\log _{4}^{N}\right)-1 ; \quad \text { when } \log _{4}^{N} \text { is an integer } \\ 2\left(\log _{4}^{2 N}\right)-1 ; \text { when } \log _{4}^{N} \text { is not an integer }\end{array}\right.$

As we can see from Equation (4), for $m=4$, the number of stages increase 2 every time when the radix of Clos network break through the line of $4^{i}$, and $i$ is an integer. For the example shown in Fig. 4, for (a) and (b) with radix 8x8 and $16 \times 16$ respectively, then they have 3 stages for going through the whole Clos network. Once the radix break s through 16 which is $4^{2}$, as shown in (c), then the Clos has 5 stages.

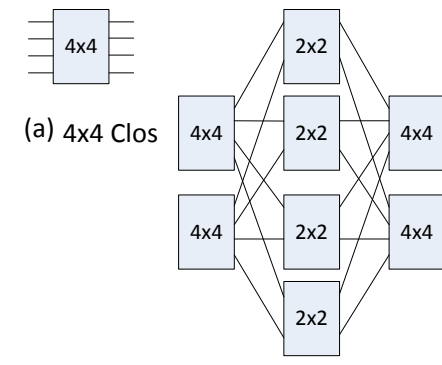

(b) $8 \times 8$ Clos

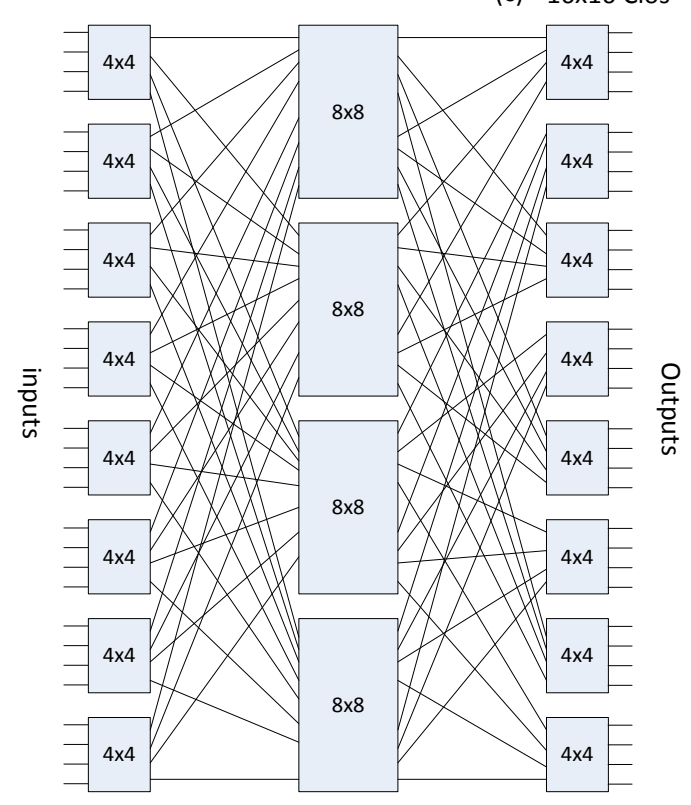

(d) $32 \times 32$ Clos

Fig. 4. Clos network

\subsection{Comparison of Clos and Benes Networks}

Comparing Equations (1) and (3), the number of logic units of Clos networks is about half of the number of logic units used in Benes networks. Notice that the logic unit represents different size crossbar switches in these two networks. As shown in Fig. 5(a) and (b), the $2 \times 2$ crossbar is made of 4 configurable switches, while the $4 \times 4$ crossbar is made of 16 configurable switches. Table 1 lists the number of logic units and configurable switches for different sized Benes and Clos networks.

Table 1 Number of logic units and configurable switches for Benes and Clos networks

\begin{tabular}{|c|c|c|c|c|}
\hline \multirow{2}{*}{ Size } & \multicolumn{2}{|c|}{ Clos } & \multicolumn{2}{c|}{ Benes } \\
\cline { 2 - 5 } & $\begin{array}{c}\text { Num of } \\
\text { logic units }\end{array}$ & $\begin{array}{c}\text { Num of } \\
\text { configurable } \\
\text { switches }\end{array}$ & $\begin{array}{c}\text { Num of } \\
\text { logic } \\
\text { units }\end{array}$ & $\begin{array}{c}\text { Num of } \\
\text { configurable } \\
\text { switches }\end{array}$ \\
\hline $4 \times 4$ & 1 & 16 & 6 & 24 \\
\hline $8 \times 8$ & 8 & 80 & 20 & 80 \\
\hline $16 \times 16$ & 12 & 192 & 56 & 224 \\
\hline $32 \times 32$ & 48 & 576 & 144 & 576 \\
\hline $64 \times 64$ & 80 & 1280 & 352 & 1408 \\
\hline $128 \times 128$ & 256 & 3328 & 832 & 3328 \\
\hline $256 \times 256$ & 448 & 7168 & 1920 & 7680 \\
\hline
\end{tabular}


Table 2 Number of Stages for Bnenes and Clos networks

\begin{tabular}{|c|c|c|}
\hline Size & Clos & Benes \\
\hline $4 \times 4$ & 1 & 3 \\
\hline $8 \times 8$ & 3 & 7 \\
\hline $16 \times 16$ & 3 & 9 \\
\hline $32 \times 32$ & 5 & 11 \\
\hline $64 \times 64$ & 5 & 13 \\
\hline $128 \times 128$ & 7 & 15 \\
\hline $256 \times 256$ & 7 & 17 \\
\hline
\end{tabular}

Equations (2) and (4) show the number of stages needed is reduced from $O\left(\log _{2}^{N}\right)$ in Benes networks to $O\left(\log _{4}^{N}\right)$ in Clos networks, when $N$ gets larger, this difference is more significant as shown in Table 2.

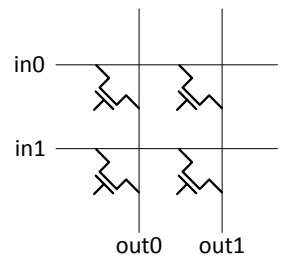

(a) $2 \times 2$ Crossbar

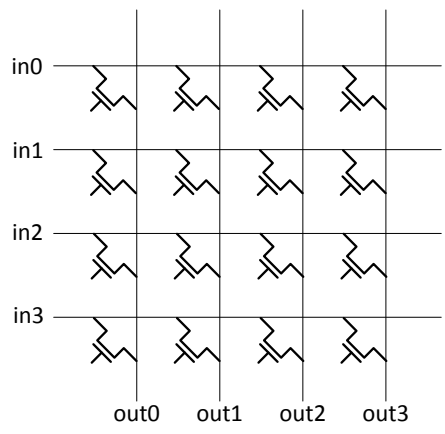

(b) $4 \times 4$ Crossbar
Fig. 5. $2 \times 2$ and $4 \times 4$ crossbar switches

As we know, the number of stages traversed by a signal is the determining factor of the timing delay. For both $2 \times 2$ and $4 \times 4$ crossbar switches, as shown in Fig. 5, only one logic unit will be passed through from an input to an output. The timing delay for the two crossbar switches should be similar. As such, the total delay experienced from an input port to an output port in Clos shall be much smaller than that in the same size Benes network.

\section{Circuit Design of Benes and Clos Network}

\subsection{Design Flow}

The circuits of different sized Benes and Clos networks are designed and simulated through the Cadence design flow, provided by their IC design tools. Circuit level layout and simulations are conducted using Cadence Virtuoso under TSMC $45 \mathrm{~nm}$ technology. The performance metrics to be compared include critical path timing delay, area, and power consumption.

Fig. 6 illustrates the design flow, which consists of three major steps: schematic design, circuit layout, and simulation. For each specific size Benes/Clos network, the schematic circuit is designed first. After the functional verification for schematic circuit is passed, the layout for each network circuit is drawn based on the schematic circuit. During the layout design period, the DRC checking need be done repeatedly in order to avoid any DRC rule violation.

After the layout is completed without any DRC violation, the net list with parasitic parameters is extracted from layout.
The LVS checking is to ensure the exact matching of the net lists generated by the schematic circuit and the layout circuit.

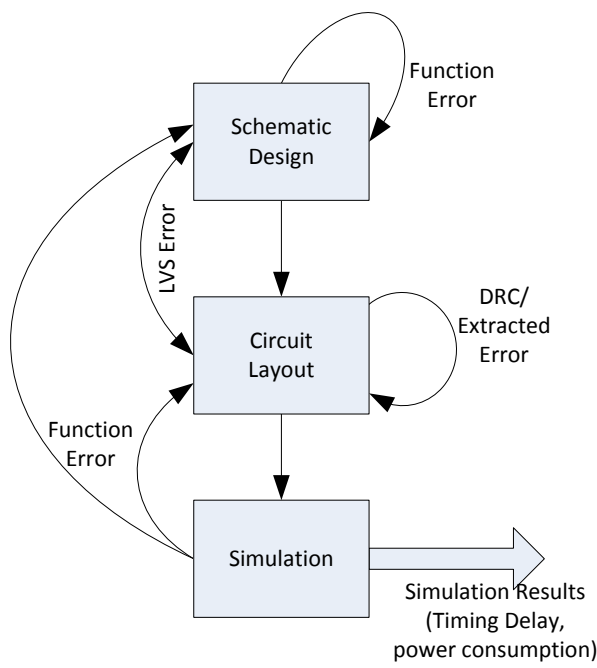

Fig. 6. Design flow

The final step is to simulate the circuits. First the simulation platform need be built using "config" view in Virtuoso. Then the tools embedded in Virtuoso are used to simulate the circuit and generate the timing delay and power consumption.

\subsection{Design of Logic Unit}

As described in Section 2, the logic unit of a Benes/Clos network is made of 4 or 16 configurable switches. We consider two alternative designs for the basic configurable switch: 1) single NMOS transistor and 2) full transmission gate which uses two transistors (one NMOS and one PMOS).

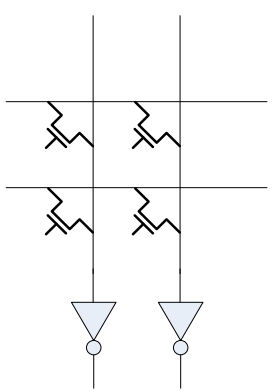

(a) Schematic View

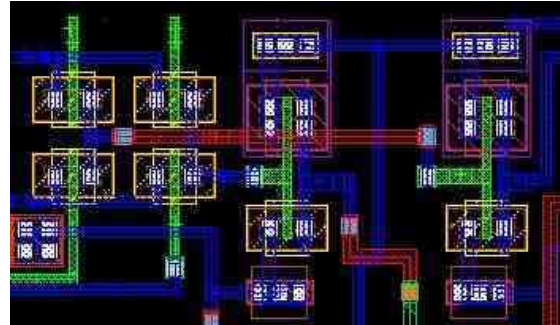

(b) Layout View
Fig. 7. NMOS-based $2 \times 2$ crossbar

Fig. 7(a) and Fig. 8(a) show the schematic diagrams of the two designs of the logic unit of $2 \times 2$ crossbar. The number of transistors needed by the first design and the second design is 6 and 16 respectively. But the NMOS transistor has its intrinsic defect known as the "weak 1" problem. The signal passes through the NMOS transistor cannot reach the VDD voltage without the help of a buffer. The transmission gate design uses the complementary manner to control the two transistors turn on or off as shown in Fig. 8. 
Both transistors are either on or off at the same time to pass or block the signal. When the input signal is ' 1 ' (' 0 '), the PMOS transistor will compensate the weak ' 1 ' (' 0 ') from the NMOS transistor.

Our experiment shows that under the TSMC $45 \mathrm{~nm}$ technology, after the signal passes through the first NMOS transistor stage, the strength of the signal can only reach $75 \%$ of the original voltage VDD. In order to solve this problem, buffers are added between every two adjacent stages so that the signal strength of all inputs for next stage is kept at VDD, as shown in Fig. 7(a) and (b). The buffers combined with slow rising of signal coming out from the NMOS transistor introduce significant delay for the signal path.

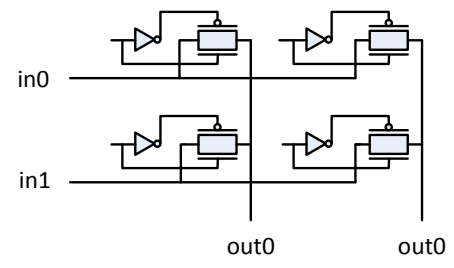

(a) Schematic View

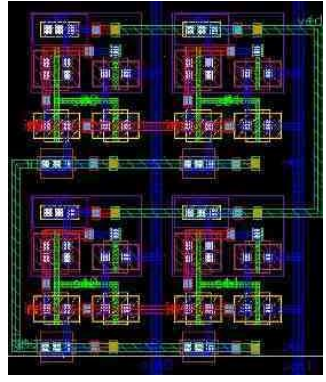

(b) Layout View
Fig. 8. TG-Based 2x2 Crossbar

\subsection{Schematic and layout Designs}

Based on the logic unit design, the schematic circuits of Benes networks are built recursively from $4 \times 4$ to $64 \times 64$ following Fig. 2. Both schematic and layout circuits are laid out manually. In this work, we have completed the layout of NMOS-based Benes networks to $32 \times 32$ and TG-based Benes networks to $64 \times 64$. The simulation results on timing delay and power consumption are generated.

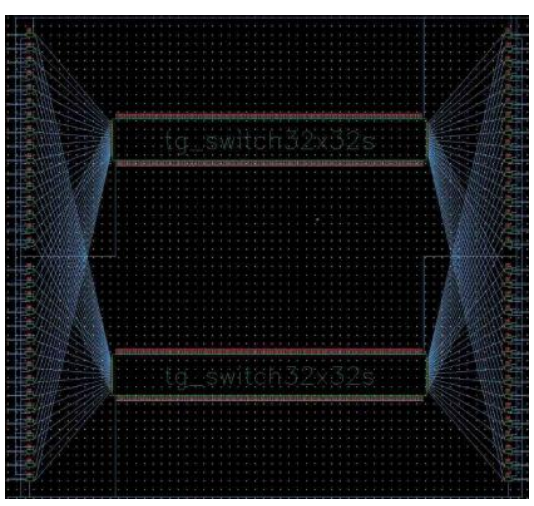

(a) Schematic View

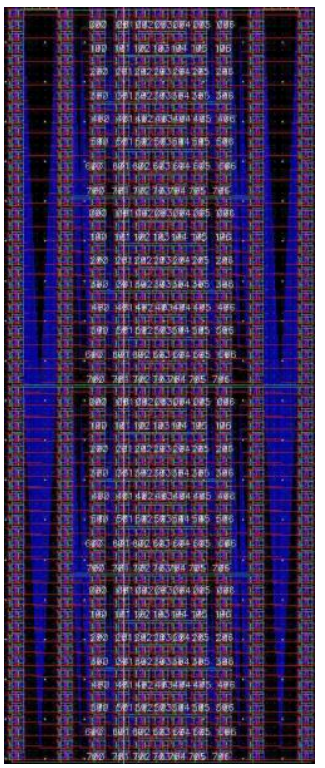

(b) Layout View
Fig. 9. Circuits of $64 \times 64$ Benes network
Fig. 9 (a) and (b) show the schematic and layout views of TG-based 64x64 Benes network as an example. When the network size is doubled, the Benes network is duplicated and added with two more stages of crossbar switches. As shown in Fig. 9(a), the $64 \times 64$ Benes network includes two 32x32 Benes networks and two input/output stages composed of thirty-two $2 \times 2$ crossbar switches.

In the similar way, the schematic and layout circuits of $4 \times 4$ to $64 \times 64$ TG-based Clos networks are laid out manually. Fig. 10 shows the circuits of $64 \times 64$ Clos network.

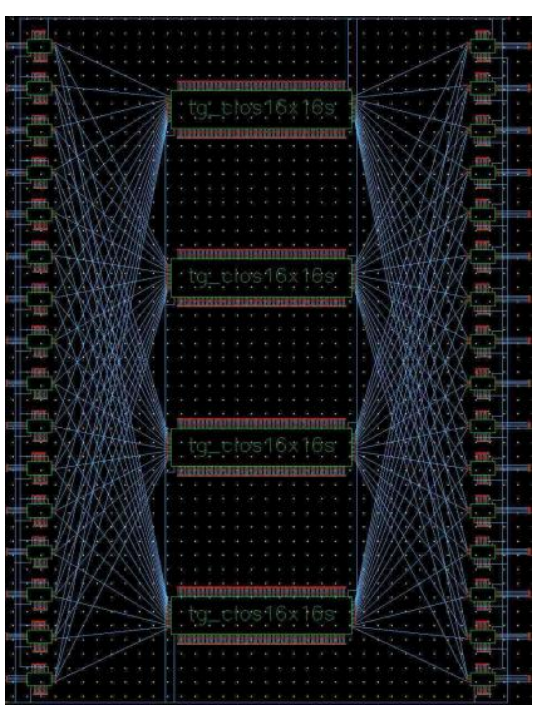

(a) Schematic View

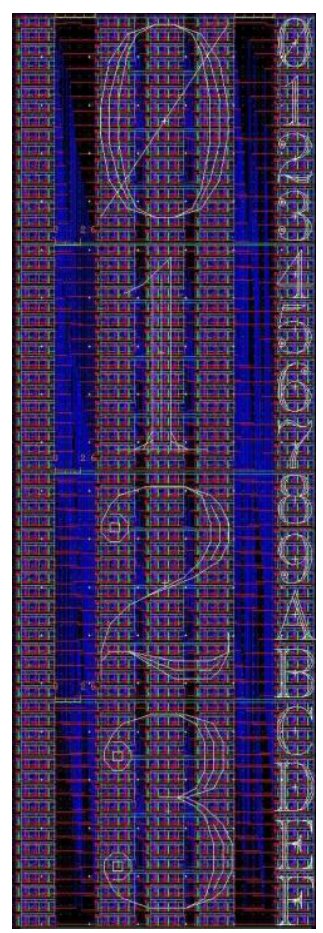

(b) Layout View
Fig. 10. Circuits of $64 \times 64$ Clos network

\section{Experimental Results}

In this section, we present the simulation results of all Benes and Clos networks obtained from Cadence Virtuoso simulation tools. The performance metrics including timing delay, area and power consumption are reported and analyzed. For timing delay and power consumption metrics, the results are obtained with wire delay model (i.e., RC model).

\subsection{Timing Delay}

Table 3 and Fig. 11 show the timing delay results of NMOS-based and TG-based Benes networks as well as TGbased Clos networks. The delay result shown is the average of the rising-transition and falling-transition delays. The wire delay has a significant impact to the timing delay. And the impact is bigger for larger size designs. This trend is attributed to factors of longer wires for interconnecting the inner and outer stages and increased wire load. As shown in the first two columns, for the same size Benes network, the delay of NMOS-based Benes network is about 10 times of the delay with its TG-based counterpart. The basic reason has been mentioned in Section 4.2. The rising delay of NMOS 
transistors along the signal path contributes the most to total path delay. In the design with TGs, the rising delay problem is eliminated, thus the path delay plummets.

Table 3 Timing delay of Benes and Clos networks

\begin{tabular}{|c|c|c|c|}
\hline $\begin{array}{c}\text { Size } \\
(\mathbf{N} \times \mathbf{N})\end{array}$ & $\begin{array}{c}\text { Benes (NMOS) } \\
(\mathbf{p s})\end{array}$ & $\begin{array}{c}\text { Benes }(\mathbf{T G}) \\
(\mathbf{p s})\end{array}$ & $\begin{array}{c}\text { Clos } \\
(\mathbf{p s})\end{array}$ \\
\hline $4 \times 4$ & 1622.83 & 101.435 & 13.615 \\
\hline $8 \times 8$ & 3131.015 & 331.545 & 192.5 \\
\hline $16 \times 16$ & 4578.965 & 625.86 & 241.84 \\
\hline $32 \times 32$ & 7034.66 & 1232.44 & 832.84 \\
\hline $64 \times 64$ & N/A & 1584.6 & 920.95 \\
\hline
\end{tabular}

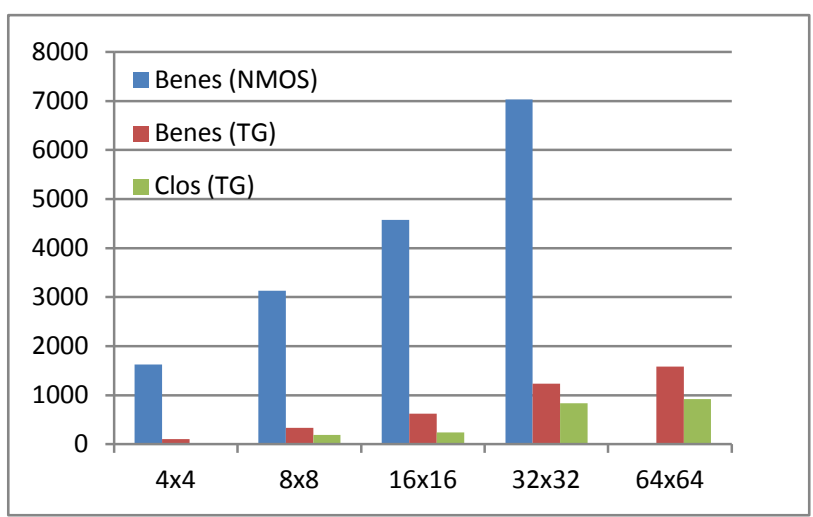

Fig. 11. Timing delay (unit: $p s$ )

The difference of timing delays between Benes and Clos networks is mostly attributed to the difference between the numbers of stages of these two networks. Table 2 shows the number of stages used for specific size networks, $8 \times 8$ and $16 \times 16$ Clos networks have as the same number of stages as $4 \times 4$ Benes networks. As shown in Fig. 11, the timing delay of $16 \times 16$ Clos is larger than that of $8 \times 8$ Clos, and both delays are higher than the timing delay of $4 \times 4$ Benes network. The reason is that though the number of stages is the major factor determining timing delay, the output load also plays an important role in it. The output load of 16x16 Clos is larger than $8 \times 8$ Clos, and both are much larger than $4 \times 4$ Benes network. This explains the trend of timing results.

\subsection{Area}

Table 4 and Table 5 show the transistor number and area of Benes and Clos networks. The first two columns show that the area of NMOS-based Benes network is much smaller than that of the corresponding TG-based Benes network. Each TG contains four transistors, which is 4 times of NMOS, as explained in Section 4.2.

Table 4 Number of Transistors used in Benes and Clos networks

\begin{tabular}{|c|c|c|c|}
\hline \multirow{2}{*}{$\begin{array}{c}\text { Size } \\
(\mathbf{N} \times \mathbf{N})\end{array}$} & Benes (NMOS) & Benes (TG) & Clos (TG) \\
\cline { 2 - 4 } & $\begin{array}{c}\text { Num of } \\
\text { Trans. }\end{array}$ & $\begin{array}{c}\text { Num of } \\
\text { Trans. }\end{array}$ & $\begin{array}{c}\text { Num of } \\
\text { Trans. }\end{array}$ \\
\hline $4 \times 4$ & 40 & 96 & 64 \\
\hline $8 \times 8$ & 176 & 320 & 320 \\
\hline $16 \times 16$ & 540 & 896 & 768 \\
\hline $32 \times 32$ & 1464 & 2304 & 2304 \\
\hline $64 \times 64$ & 3696 & 5632 & 5120 \\
\hline
\end{tabular}

Table 5 Area of Benes and Clos networks

\begin{tabular}{|c|c|c|c|}
\hline \multirow{2}{*}{$\begin{array}{c}\text { Size } \\
(\mathbf{N} \times \mathbf{N})\end{array}$} & Benes (NMOS) & Benes (TG) & Clos (TG) \\
\cline { 2 - 4 } & $\begin{array}{c}\text { Area } \\
\left(\boldsymbol{u m}^{2}\right)\end{array}$ & $\begin{array}{c}\text { Area } \\
\left(\boldsymbol{u m}^{2}\right)\end{array}$ & $\begin{array}{c}\text { Area } \\
\left(\boldsymbol{u m}^{2}\right)\end{array}$ \\
\hline $4 \times 4$ & 40.97 & 75.81 & 42.56 \\
\hline $8 \times 8$ & 154.10 & 263.05 & 227.25 \\
\hline $16 \times 16$ & 474.42 & 764.71 & 624.03 \\
\hline $32 \times 32$ & 684.13 & 1239.2 & 1125.7 \\
\hline $64 \times 64$ & N/A & 3662.8 & 2954.1 \\
\hline
\end{tabular}

While NMOS-based Benes networks also need adding the inverters (as buffers) between two neighboring stages to reshape the defect signals caused by weak ' 1 ' problem. As such, the transistor account of a TG-based Benes network is slightly more than 2 times of its NMOS-based counterpart. The actual area ration between TG- and NMOS-based Benes networks is less than 2:1.

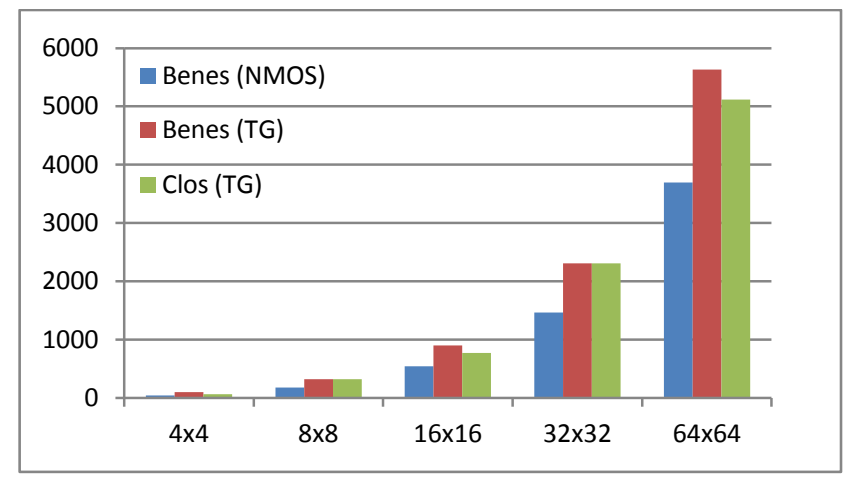

Fig. 12. Number of transistors

Fig. 12 shows the trend described in Table 4, as we can see, the TG-based Benes and Clos consume similar amount of transistors, which is higher than NMOS-based network. As shown in Fig. 13, consistent with the trend of transistor count, the area of NMOS-based Benes network is the smallest among the three designs. The TG-based Clos network has smaller area than TG-based Benes network for all network sizes and the difference is more significant for larger size $N$. The smaller layout area of Clos networks is attributed to the fact that by using $4 \times 4$ crossbars as major building blocks, less interconnects are used and the circuit is more compacted compared with that of Benes networks.

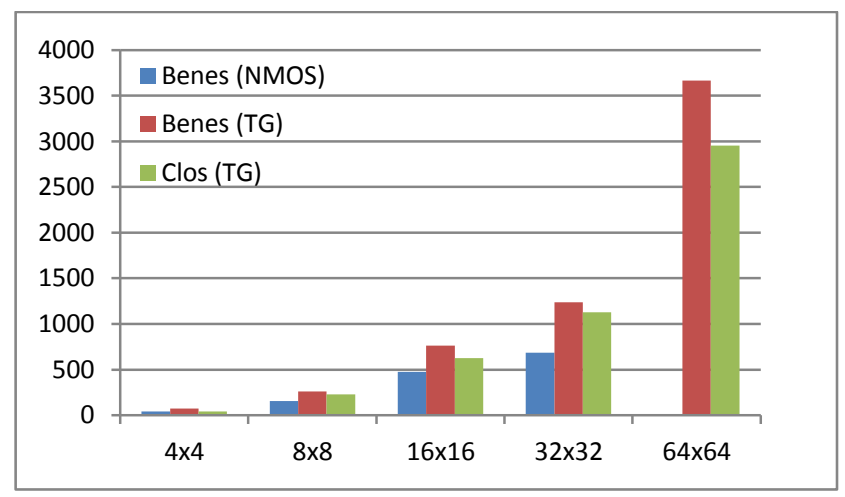

Fig. 13. Area (unit: $u m^{2}$ ) 


\subsection{Power Consumption}

Table 6 shows the power consumption for these networks. The results with $\mathrm{RC}$ model are much higher than without $\mathrm{RC}$ model. Similar to timing delay results, the impact of wire delay is getting bigger with the network size increasing. The power consumption of NMOS-based Benes network is higher than the corresponding TG-based Benes network, because the inverters between two neighboring stages consume significant power to compensate the defect signals caused by NMOS transistor's weak ' 1 ' problem.

Table 6 Power consumption of Benes and Clos networks

\begin{tabular}{|c|c|c|c|}
\hline $\begin{array}{c}\text { Size } \\
(\mathbf{N} \times \mathbf{N})\end{array}$ & $\begin{array}{c}\text { Benes (NMOS) } \\
(\mathbf{w})\end{array}$ & $\begin{array}{c}\text { Benes (TG) } \\
(\mathbf{w})\end{array}$ & $\begin{array}{c}\text { Clos (TG) } \\
(\mathbf{w})\end{array}$ \\
\hline $4 \times 4$ & $1.149 \mathrm{E}-6$ & $0.9278 \mathrm{E}-6$ & $2.748 \mathrm{E}-7$ \\
\hline $8 \times 8$ & $4.162 \mathrm{E}-6$ & $2.491 \mathrm{E}-6$ & $2.084 \mathrm{E}-6$ \\
\hline $16 \times 16$ & $8.064 \mathrm{E}-6$ & $7.954 \mathrm{E}-6$ & $3.282 \mathrm{E}-6$ \\
\hline $32 \times 32$ & $16.23 \mathrm{E}-6$ & $10.45 \mathrm{E}-6$ & $8.415 \mathrm{E}-6$ \\
\hline $64 \times 64$ & N/A & $32.85 \mathrm{E}-6$ & $25.7 \mathrm{E}-6$ \\
\hline
\end{tabular}

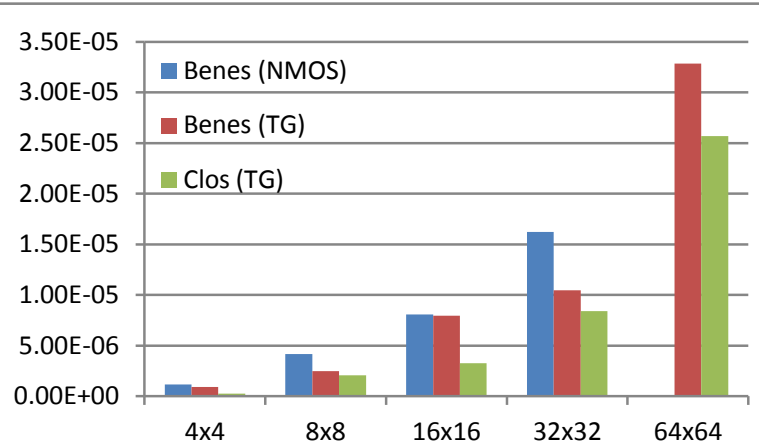

Fig. 14. Power consumption (unit: $w$ )

Fig. 14 shows that comparing TG-based Benes and Clos networks, the Benes networks consume more power. On one hand, the data signals go through more stages in Benes network than in Clos network, on the other hand, the signals in Clos network have larger output load considering the larger size logic unit. The combined effect is that Clos networks have lower power consumption than Benes networks. The improvement (over 20\%) is more significant for larger size $N$ as shown in Fig. 14.

\section{Conclusion}

This paper is focused on the circuit designs of different sized Benes and Clos networks considering two types of implementations: with NMOS transistors and full transmission gates. All designs are laid out manually and simulated with Cadence tools. The experimental results show that the TGbased Benes networks have much better timing and power performance than NMOS-based counterparts, though more transistor resources are needed in TG-based designs. Clos networks have $60 \%$ timing delay reduction than Benes networks with even smaller area and power consumption. This result confirms that Clos network is a better alternative than Benes networks to replace crossbars in large scale networks. One of the future work is to explore the network performance of Benes/Clos-based NoCs under both synthetic and real life applications.

\section{References}

[1] P. Kundu, On-die interconnects for next generation CMPs, in: Proc. Workshop On- and Off-Chip Interconnection Networks for Multicore Systems (OCIN), 2006.

[2] C. O. Chen, S. Park, T. Krishna, L. Peh, A low-swing crossbar and link generator for low-power network-onchi, in: Proc. IEEE/ACM Int'l Conf. Computer-Aided Design (ICCAD), 2011, pp. 779-786.

[3] R. Das, S. Eachempati, A. K. Mishra, V. Narayanan, and C. R. Das, Design and evaluation of a hierachical on-chip interconnect for next-generation CMPs, in: Proc. IEEE 15th Symp. HPCA, 2009, pp. 175-186.

[4] X. Wang, M. Yang, Y. Jiang, and P. Liu, A power-aware mapping approach to map IP cores onto NoCs under bandwidth and latency constraints, ACM Trans. Architecture and Code Optimization, 7 (2010).

[5] D.Wentzlaff and P. Griffin, On-chip interconnection architecture of the tile processor, IEEE Micro, 27 (2007), 15-31.

[6] C. Kim, D. Burger, S. W. Keckler, Nonuniform cache architectures for wire delay dominated on-chip caches, IEEE Micro, 23 (2003), 99-107.

[7] T. Johnson and U. Nawathe, An 8-core, 64-thread, 64-bit power efficient SPARC SOC (niagara2), in: Proc. IEEE SSCC, 2007, pp. 108-590.

[8] L. Mhamdi, K. Goossens, I.V. Senin., Buffered crossbar fabrics based on networks on chip, in: Proc. Communication Networks and Services Research Conference (CNSR), 2010, pp.74-79.

[9] G. Passas, M. Katevenis, D. Pnevmatikatos, A 128 x 128 x 24Gb/s crossbar interconnecting 128 tiles in a single hop and occupying $6 \%$ of their area, in: Proc. $4^{\text {th }}$ ACM/IEEE Int'1 Symp. Network-on-Chip (NoCS) , 2010, pp. 87-95.

[10] J. Kim, J. Dally, B. Towles, A. K. Gupta, Microarchitecture of a high-radix router, in: Proc. 32nd Int't Sym. Computer Architecture, 2005.

[11] J. Duato, I. Johnson, J. Flich, F. Naven, P. Garcia, and T. Nachiondo, A new scalable and cost-effective congestion management strategy for lossless multistage interconnection networks, in: Proc. Int'l Symp. HPCA, 2005.

[12] G. Mora, J. Flich, J. Duato, P. L'opez, E. Baydal, and O. Lysne, Towards an efficient switch architecture for highradix switches, in: Proc. ACM/IEEE ANCS, 2006.

[13] R. Haring, The IBM blue gene/q compute chip+simd floating-point unit, in: Proc. Symp. High-Performance Chips, 2011.

[14] K. Sewell, R.G. Dreslinski, T. Manville, S. Satpathy, N. Pinckney, G. Blake, M. Cieslak, et al., Swizzle-switch networks for many-core systems, IEEE J. Emerging and Selected Topics in Circuits and Systems, 2 (2012), 278294. 
[15] C. Kim, D. Burger, and S. W. Keckler, An adaptive, nonuniform cache structure for wire-delay dominated on-chip caches, in: Proc. 10th Int'l Conf. Architectural Support Programm. Languages Operat. Syst., 2002, pp. 211-222.

[16] A. Zia, S. Kannan, G. Rose and H. J. Chao, Highlyscalable 3D Clos NoC for many-core CMPs, in: Proc. IEEE 8th Int'l NEWCAS Conf. (NEWCAS), 2010, pp. 229-232.

[17] E. Coen-Alfaro, Crossbar architectures for VLSI systems: a comparative study, Master Thesis, University of Idaho, Apr. 2004.

[18] Y. Zhang, J. Taikyeong, F. Chen, H. Wu, and N. R. Gao, A study of the on-chip interconnection network for the IBM Cyclops64 multi-core architecture, in: Proc. Parallel and Distributed Processing Symp. (IPDPS), 2006, pp. 2529.

[19] Y. Kao, M. Yang, N.S. Artan, and H. J. Chao, CNoC: High-radix Clos network-on-chip, IEEE Trans. Computer-Aided Design of Integrated Circuits and Systems, 30 (2011), 1897-1910.

[20] D. L. Lewis, S. Yalamanchili, and H. S. lee, High performance non-blocking switch design in 3D diestacking technology, in: Proc. IEEE Computer Society Annual Symposium on VLSI, 2009.

[21] C. Clos, A study of non-blocking switching networks, Bell System Technical Journal, 32 (1952), 406424.

[22] G. Passas, M. Katevenis, D. Pnevmatikatos, VLSI microarchitectures for high-radix crossbar schedulers, in: Proc. 5th IEEE/ACM Int. Symp. Networks Chip, 2011, pp. 217224.

[23] C. Kim, D. Burger, S. Keckler, An Adaptive, Nonuniform cache structure for wire-delay dominated on-chip caches, in: Proc. $10^{\text {th }}$ Int'l Conf. ASPLOS, 2002, 211222.

[24] S. Satpathy, Z. Foo, B. Giridhar, R. Dreslinski, D. Sylvester, T. Mudge, D. Blaauw, A 1.07Tbit/s 128×128 swizzle network for SIMD processors, in: Proc. IEEE Symp. VLSI Circuits, Jun. 2010, 16-18.

[25] S. Satpathy, K. Sewell, T. Manville, Y. Chen, R. Dreslinski, D. Sylvester, T. Mudge, D. Blaauw, A 4.5Tb/s $3.4 \mathrm{~Tb} / \mathrm{s} / \mathrm{W} 64 \times 64$ switch fabric with self-updating leastrecently-granted priority and quality-of-service arbitration in 45nm CMOS, in: Proc. IEEE ISSCC, Feb. 2012, 478480.

[26] H. Moussa, O. Muller, A. Baghdadi, M. Jezequel, Butterfly and Benes-based on-chip communication networks for multiprocessor turbo decoding, in: Proc. Design, Automation \& Test in Europe Conference \& Exhibition, 2007, 1-6.

[27] A. Abbas, M. Ali, A. Fayyaz, A. Ghosh, A. Kalra, S. U. Khan, A survey on envergy-efficient methodologies and architectures of network-on-chip, in: Proc. Computers \& Electrical Engineering., 40 (2014), 333-347.

[28] H. Liu, L. Xie, J. Liu and L. Ding, Application of butterfly Clos-network in network-on-chip in: Proc. Scientific World Journal, (2014), 1-11.
[29] H. Richer, Real-time interconnection network for singlechip many-core computers, in: Proc. Design \& Test Symposium 2013 East-West, 2013, 1-4.

[30] Y. Kao and H. J. Chao, Design of a bufferless photonic Clos network-on-chip architecture, in: Proc. IEEE Transactions on Computers, 63 (2014), 764-776. 